\title{
On Relation between Porosity of Epitaxial Layer and Quantity of Radiation Defects Generated during Radiation Processing in a Multilayer Structure
}

\author{
E. L. Pankratov ${ }^{1}$ and E. A. Bulaeva ${ }^{1,2}$ \\ ${ }^{1}$ Nizhny Novgorod State University, 23 Gagarin Avenue, Nizhny Novgorod 603950, Russia \\ ${ }^{2}$ Nizhny Novgorod State University of Architecture and Civil Engineering, 65 Il'insky Street, Nizhny Novgorod 603950, Russia
}

Correspondence should be addressed to E. L. Pankratov; elp2004@mail.ru

Received 16 March 2016; Revised 5 May 2016; Accepted 25 May 2016

Academic Editor: Oleg Lupan

Copyright (C) 2016 E. L. Pankratov and E. A. Bulaeva. This is an open access article distributed under the Creative Commons Attribution License, which permits unrestricted use, distribution, and reproduction in any medium, provided the original work is properly cited.

We analyzed redistribution of radiation defects in a multilayer structure with porous epitaxial layer. The radiation defects were generated during radiation processing. It has been shown that porosity of epitaxial layer gives a possibility to decrease quantity of radiation defects.

\section{Introduction}

One of actual questions of solid state electronics is increasing their radiation resistance. The increasing gives a possibility to decrease influence of different types of irradiation on characteristics of solid state electronic devices. Several methods have been used to increase the radiation resistance of devices of solid state electronics [1-5]. One way to solve the problem is using an epitaxial layer over considered devices (overlayer) to organize the resistance. Another way to increase the radiation resistance is using special epitaxial layers under considered devices to use these layers as drain of radiation defects. An alternative approach to the considered one is using porous materials near device area to use the porosity as drain of radiation defects again. In this paper, we consider an approach to decreasing quantity of radiation defects. The radiation defects have been generated during radiation processing of materials. For the framework the approach, we consider a heterostructure. The heterostructure consists of a substrate and porous epitaxial layer (see Figure 1). We assume that the substrate was under the influence of radiation processing (ion implantation, cosmic radiation etc.) through the epitaxial layer. Radiation processing of materials leads to generation of radiation defects. Main aim of the present paper is analysis of influence of porosity of epitaxial layer on distribution of concentration of radiation defects in the considered heterostructure.

\section{Method of Solution}

To solve our aim, we calculate distributions of concentrations of radiation defects in considered heterostructure in space and time. We determine the above distributions as solutions of the following system of equations [6-12]:

$$
\begin{aligned}
& \frac{\partial I(x, y, z, t)}{\partial t} \\
& =\frac{\partial}{\partial x}\left[D_{I}(x, y, z, T) \frac{\partial I(x, y, z, t)}{\partial x}\right] \\
& \quad+\frac{\partial}{\partial y}\left[D_{I}(x, y, z, T) \frac{\partial I(x, y, z, t)}{\partial y}\right] \\
& \quad+\frac{\partial}{\partial z}\left[D_{I}(x, y, z, T) \frac{\partial I(x, y, z, t)}{\partial z}\right] \\
& \quad-k_{I, V}(x, y, z, T) I(x, y, z, t) V(x, y, z, t)
\end{aligned}
$$




$$
\begin{aligned}
& +\frac{\partial}{\partial x}\left[\frac{D_{I S}}{\bar{V} k T} \frac{\partial \mu(x, y, z, t)}{\partial x}\right] \\
& +\frac{\partial}{\partial y}\left[\frac{D_{I S}}{\bar{V} k T} \frac{\partial \mu(x, y, z, t)}{\partial y}\right] \\
& +\frac{\partial}{\partial z}\left[\frac{D_{I S}}{\bar{V} k T} \frac{\partial \mu(x, y, z, t)}{\partial z}\right] \\
& -k_{I, I}(x, y, z, T) I^{2}(x, y, z, t), \\
& \frac{\partial V(x, y, z, t)}{\partial t} \\
& =\frac{\partial}{\partial x}\left[D_{V}(x, y, z, T) \frac{\partial V(x, y, z, t)}{\partial x}\right] \\
& +\frac{\partial}{\partial y}\left[D_{V}(x, y, z, T) \frac{\partial V(x, y, z, t)}{\partial y}\right] \\
& +\frac{\partial}{\partial z}\left[D_{V}(x, y, z, T) \frac{\partial V(x, y, z, t)}{\partial z}\right] \\
& -k_{I, V}(x, y, z, T) I(x, y, z, t) V(x, y, z, t) \\
& +\frac{\partial}{\partial x}\left[\frac{D_{V S}}{\bar{V} k T} \frac{\partial \mu(x, y, z, t)}{\partial x}\right] \\
& +\frac{\partial}{\partial y}\left[\frac{D_{V S}}{\bar{V} k T} \frac{\partial \mu(x, y, z, t)}{\partial y}\right] \\
& +\frac{\partial}{\partial z}\left[\frac{D_{V S}}{\bar{V} k T} \frac{\partial \mu(x, y, z, t)}{\partial z}\right] \\
& -k_{V, V}(x, y, z, T) V^{2}(x, y, z, t) \text {. }
\end{aligned}
$$

Here $I(x, y, z, t)$ and $V(x, y, z, t)$ are the distributions of concentrations of radiation interstitials and vacancies in space and time, respectively. The first, the second, and the third terms of both equations describe diffusion of point defects with the diffusion coefficients $D_{I}(x, y, z, T)$ for interstitials and $D_{V}(x, y, z, T)$ and vacancies, respectively. The fourth terms of both equations describe recombination of point defects with the parameter of recombination $k_{I, V}(x, y, z, T)$. The fifth, sixth, and seventh terms of both equations describe correction to diffusion due to porosity of material. The functions $D_{I S}(x, y, z, T)$ and $D_{V S}(x, y, z, T)$ describe dependencies of diffusion coefficients of defects due to porosity of materials on coordinate and temperature $T ; k$ is the Boltzmann constant; $\bar{V}$ is the molar volume; $\mu(x, y, z, T)=R$. $T \cdot \ln \left(V_{2} / V_{1}\right)[10]$ is the chemical potential; $V_{1} u V_{2}$ are the initial and final volume of pores, respectively; $R=8.31 \mathrm{~J} /($ mole $\cdot \mathrm{K}$ ) is the molar gas constant. Last terms of (1) with nonlinearity of concentrations of defects $V^{2}(x, y, z, t)$ and $I^{2}(x, y, z, t)$ correspond to generation of divacancies and analogous complexes of interstitials (see, e.g., [11] and appropriate references in this work). The functions $k_{I, I}(x, y, z, T)$ and $k_{V, V}(x, y, z, T)$

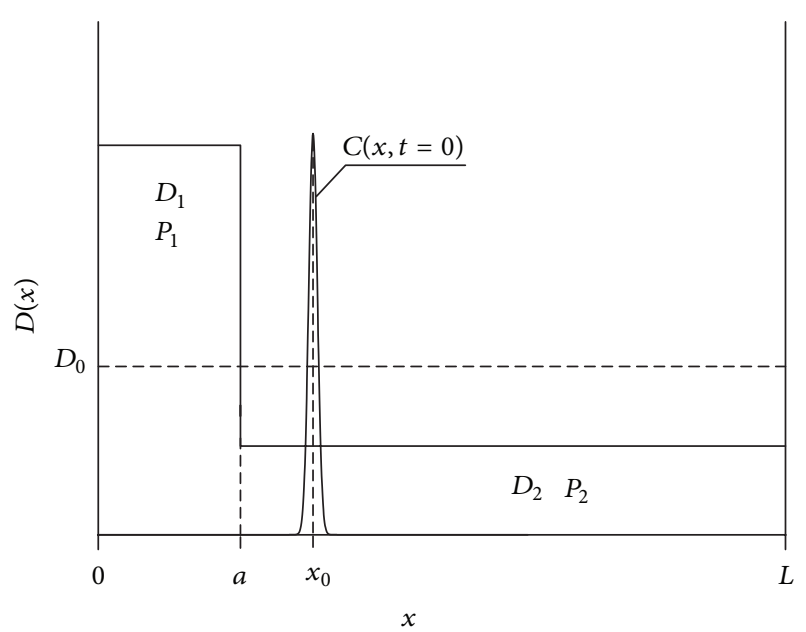

FIgURE 1: Heterostructure, which consists of a substrate and an epitaxial layer. The figure also shows distribution of concentration of implanted dopant.

describe dependencies of the parameters of generation of complexes point defects.

Boundary and initial conditions for (1) could be written as

$\left.\frac{\partial I(x, y, z, t)}{\partial x}\right|_{x=0}=0$,
$\left.\frac{\partial I(x, y, z, t)}{\partial x}\right|_{x=L_{x}}=0$,

$\left.\frac{\partial I(x, y, z, t)}{\partial y}\right|_{y=0}=0$,

$\left.\frac{\partial I(x, y, z, t)}{\partial y}\right|_{y=L_{y}}=0$,

$\left.\frac{\partial I(x, y, z, t)}{\partial z}\right|_{z=0}=0$,

$\left.\frac{\partial I(x, y, z, t)}{\partial z}\right|_{z=L_{z}}=0$,

$\left.\frac{\partial V(x, y, z, t)}{\partial x}\right|_{x=0}=0$,

$\left.\frac{\partial V(x, y, z, t)}{\partial x}\right|_{x=L_{x}}=0$,

$\left.\frac{\partial V(x, y, z, t)}{\partial y}\right|_{y=0}=0$,

$\left.\frac{\partial V(x, y, z, t)}{\partial y}\right|_{y=L_{y}}=0$ 


$$
\begin{aligned}
& \left.\frac{\partial V(x, y, z, t)}{\partial z}\right|_{z=0}=0, \\
& \left.\frac{\partial V(x, y, z, t)}{\partial z}\right|_{z=L_{z}}=0, \\
& I(x, y, z, 0)=f_{I}(x, y, z), \\
& V(x, y, z, 0)=f_{V}(x, y, z), \\
& V\left(x,+V_{n} t, y_{1}+V_{n} t, z_{1}+V_{n} t, t\right) \\
& \quad=V^{*}\left(1+\frac{2 \ell \omega}{k T \sqrt{x_{1}^{2}+y_{1}^{2}+z_{1}^{2}}}\right) .
\end{aligned}
$$

Here $I$ and $V^{*}$ are the equilibrium distributions of concentrations of interstitials and vacancies, respectively; $\omega=a^{3}$, $a$ is the atomic spacing; $\ell$ is the specific surface energy. The above boundary conditions correspond to absence of flow of point defects through external boundary of heterostructure and absorption of these defects by pores (last condition). The above initial conditions correspond to distributions of concentration of the above defects after finishing radiation processing. To take into account porosity, we assume, that porous are approximately cylindrical with average dimensions $r=\sqrt{x_{1}^{2}+y_{1}^{2}}$ and $z_{1}$ [13]. With time, small pores decompose into vacancies, and the vacancies are absorbed by large pores [10]. The large pores take spherical form during the absorption [10]. Distribution of concentration of vacancies, which was formed due to porosity, could be determined by summing all pores; that is,

$$
\begin{aligned}
V(x, y, z, t) \\
=\sum_{i=0}^{l} \sum_{j=0}^{m} \sum_{k=0}^{n} V_{p}(x+i \alpha, y+j \beta, z+k \chi, t), \\
R=\sqrt{x^{2}+y^{2}+z^{2}} .
\end{aligned}
$$

Here $\alpha, \beta$, and $\chi$ are averaged distances between centers of pores in $x, y$, and $z$ directions, respectively; $l, m$, and $n$ are quantities of pores in the same directions.

We determine distributions of concentrations of divacancies $\Phi_{V}(x, y, z, t)$ and diinterstitials $\Phi_{I}(x, y, z, t)$ in space and time by solving the following system of equations $[9,11,12$, 14]:

$$
\begin{aligned}
& \frac{\partial \Phi_{I}(x, y, z, t)}{\partial t} \\
& =\frac{\partial}{\partial x}\left[D_{\Phi_{I}}(x, y, z, T) \frac{\partial \Phi_{I}(x, y, z, t)}{\partial x}\right] \\
& \quad+\frac{\partial}{\partial y}\left[D_{\Phi_{I}}(x, y, z, T) \frac{\partial \Phi_{I}(x, y, z, t)}{\partial y}\right]
\end{aligned}
$$

$$
\begin{aligned}
& +\frac{\partial}{\partial z}\left[D_{\Phi_{I}}(x, y, z, T) \frac{\partial \Phi_{I}(x, y, z, t)}{\partial z}\right] \\
& +k_{I, I}(x, y, z, T) I^{2}(x, y, z, t) \\
& +k_{I}(x, y, z, T) I(x, y, z, t) \\
& +\frac{\partial}{\partial x}\left[\frac{D_{\Phi_{I} S}}{\bar{V} k T} \frac{\partial \mu(x, y, z, t)}{\partial x}\right] \\
& +\frac{\partial}{\partial y}\left[\frac{D_{\Phi_{I} S}}{\bar{V} k T} \frac{\partial \mu(x, y, z, t)}{\partial y}\right] \\
& \left.+\frac{\partial}{\partial z}\left[\frac{D_{\Phi_{I} S}}{\bar{V} k T} \frac{\partial \mu(x, y, z, t)}{\partial z}\right] \frac{\partial \Phi_{V}(x, y, z, t)}{\partial t}\right] \\
& +\frac{\partial}{\partial x}\left[D_{\Phi_{V}}(x, y, z, T) \frac{\partial \Phi_{V}(x, y, z, t)}{\partial x}\right] \\
& +\frac{\partial}{\partial y}\left[\frac{D_{\Phi_{V} S}}{\bar{V} k T} \frac{\partial \mu(x, y, z, t)}{\partial z}\left[\frac{D_{\Phi_{V} S}}{\bar{V} k T} \frac{\partial \mu(x, y, z, t)}{\partial y}\right]\right. \\
& +k_{V}(x, y, z, T) V(x, y, z, t) \\
& +\frac{\partial}{\partial z}\left[D_{\Phi_{V}}(x, y, z, T) \frac{\partial \Phi_{V}(x, y, z, t)}{\partial y}\right] \\
& \left.+\frac{D_{\Phi_{V}}}{\partial y}(x, y, z, T) \frac{\partial \Phi_{V}(x, y, z, t)}{\partial z}\right]
\end{aligned}
$$

The first, the second, and the third terms of both equations describe diffusion of point defects with the diffusion coefficients $D_{\Phi_{I}}(x, y, z, T)$ for diinterstitials and $D_{\Phi_{V}}(x, y$, $z, T)$ for divacancies. The fourth terms of both equations correspond to generation of new diinterstitials and divacancies. The fifth terms of the above equations correspond to decay of existing diinterstitials and divacancies. The functions $k_{I}(x, y, z, T)$ and $k_{V}(x, y, z, T)$ describe the parameters of decay of the above complexes on coordinate and temperature. The last terms of both equations describe correction to diffusion due to porosity of material. The functions $D_{\Phi_{I} S}(x, y, z, T)$ and $D_{\Phi_{V} S}(x, y, z, T)$ describe dependencies of diffusion coefficients of defects due to porosity materials on coordinate and temperature. Boundary and initial conditions for (4) could be written as

$$
\begin{gathered}
\left.\frac{\partial \Phi_{I}(x, y, z, t)}{\partial x}\right|_{x=0}=0, \\
\left.\frac{\partial \Phi_{I}(x, y, z, t)}{\partial x}\right|_{x=L_{x}}=0
\end{gathered}
$$




$$
\begin{aligned}
& \left.\frac{\partial \Phi_{I}(x, y, z, t)}{\partial y}\right|_{y=0}=0, \\
& \left.\frac{\partial \Phi_{I}(x, y, z, t)}{\partial y}\right|_{y=L_{y}}=0, \\
& \left.\frac{\partial \Phi_{I}(x, y, z, t)}{\partial z}\right|_{z=0}=0, \\
& \left.\frac{\partial \Phi_{I}(x, y, z, t)}{\partial z}\right|_{z=L_{z}}=0, \\
& \left.\frac{\partial \Phi_{V}(x, y, z, t)}{\partial x}\right|_{x=0}=0, \\
& \left.\frac{\partial \Phi_{V}(x, y, z, t)}{\partial x}\right|_{x=L_{x}}=0, \\
& \left.\frac{\partial \Phi_{V}(x, y, z, t)}{\partial y}\right|_{y=0}=0, \\
& \left.\frac{\partial \Phi_{V}(x, y, z, t)}{\partial y}\right|_{y=L_{y}}=0, \\
& \left.\frac{\partial \Phi_{V}(x, y, z, t)}{\partial z}\right|_{z=0}=0, \\
& \left.\frac{\partial \Phi_{V}(x, y, z, t)}{\partial z}\right|_{z=L_{z}}=0, \\
& \Phi_{I}(x, y, z, 0)=f_{\Phi_{I}}(x, y, z), \\
& =\Phi_{\Phi_{V}}(x, y, z) .
\end{aligned}
$$

The above boundary conditions correspond to absence of flow of point defects through external boundary of heterostructure. The above initial conditions correspond to distributions of concentration of the above defects after finishing radiation processing.

We determine distributions of concentrations of radiation defects in space and time by method of averaging of function corrections [14-16]. To use the approach, we write (1) and (4) on account of initial distributions of defects; that is,

$$
\begin{aligned}
& \frac{\partial I(x, y, z, t)}{\partial t} \\
& =\frac{\partial}{\partial x}\left[D_{I}(x, y, z, T) \frac{\partial I(x, y, z, t)}{\partial x}\right] \\
& \quad+\frac{\partial}{\partial y}\left[D_{I}(x, y, z, T) \frac{\partial I(x, y, z, t)}{\partial y}\right] \\
& +\frac{\partial}{\partial z}\left[D_{I}(x, y, z, T) \frac{\partial I(x, y, z, t)}{\partial z}\right] \\
& \quad-k_{I, V}(x, y, z, T) I(x, y, z, t) V(x, y, z, t)
\end{aligned}
$$

$$
\begin{aligned}
& +f_{I}(x, y, z) \delta(t)+\frac{\partial}{\partial x}\left[\frac{D_{I S}}{\bar{V} k T} \frac{\partial \mu(x, y, z, t)}{\partial x}\right] \\
& +\frac{\partial}{\partial y}\left[\frac{D_{I S}}{\bar{V} k T} \frac{\partial \mu(x, y, z, t)}{\partial y}\right] \\
& +\frac{\partial}{\partial z}\left[\frac{D_{I S}}{\bar{V} k T} \frac{\partial \mu(x, y, z, t)}{\partial z}\right] \\
& -k_{I, I}(x, y, z, T) I^{2}(x, y, z, t),
\end{aligned}
$$$$
\frac{\partial V(x, y, z, t)}{\partial t}
$$$$
=\frac{\partial}{\partial x}\left[D_{V}(x, y, z, T) \frac{\partial V(x, y, z, t)}{\partial x}\right]
$$$$
+\frac{\partial}{\partial y}\left[D_{V}(x, y, z, T) \frac{\partial V(x, y, z, t)}{\partial y}\right]
$$$$
+\frac{\partial}{\partial z}\left[D_{V}(x, y, z, T) \frac{\partial V(x, y, z, t)}{\partial z}\right]
$$$$
-k_{I, V}(x, T) I(x, y, z, t) V(x, y, z, t)
$$$$
+f_{V}(x, y, z) \delta(t)+\frac{\partial}{\partial x}\left[\frac{D_{V S}}{\bar{V} k T} \frac{\partial \mu(x, y, z, t)}{\partial x}\right]
$$$$
+\frac{\partial}{\partial y}\left[\frac{D_{V S}}{\bar{V} k T} \frac{\partial \mu(x, y, z, t)}{\partial y}\right]
$$$$
+\frac{\partial}{\partial z}\left[\frac{D_{V S}}{\bar{V} k T} \frac{\partial \mu(x, y, z, t)}{\partial z}\right]
$$$$
-k_{V, V}(x, y, z, T) V^{2}(x, y, z, t) \text {, }
$$

$$
\begin{aligned}
\frac{\partial \Phi_{I}(x, y, z, t)}{\partial t} & =\frac{\partial}{\partial x}\left[D_{\Phi_{I}}(x, y, z, T) \frac{\partial \Phi_{I}(x, y, z, t)}{\partial x}\right] \\
& +\frac{\partial}{\partial y}\left[D_{\Phi_{I}}(x, y, z, T) \frac{\partial \Phi_{I}(x, y, z, t)}{\partial y}\right] \\
& +\frac{\partial}{\partial z}\left[D_{\Phi_{I}}(x, y, z, T) \frac{\partial \Phi_{I}(x, y, z, t)}{\partial z}\right] \\
& +k_{I, I}(x, y, z, T) I^{2}(x, y, z, t) \\
& -k_{I}(x, y, z, T) I(x, y, z, t) \\
& +\frac{\partial}{\partial x}\left[\frac{D_{\Phi_{V} S}}{\bar{V} k T} \frac{\partial \mu(x, y, z, t)}{\partial x}\right] \\
& +\frac{\partial}{\partial y}\left[\frac{D_{\Phi_{V} S}}{\bar{V} k T} \frac{\partial \mu(x, y, z, t)}{\partial y}\right]
\end{aligned}
$$




$$
\begin{aligned}
& +\frac{\partial}{\partial z}\left[\frac{D_{\Phi_{V} S}}{\bar{V} k T} \frac{\partial \mu(x, y, z, t)}{\partial z}\right] \\
& +f_{\Phi_{I}}(x, y, z) \delta(t), \\
& \frac{\partial \Phi_{V}(x, y, z, t)}{\partial t} \\
& =\frac{\partial}{\partial x}\left[D_{\Phi_{V}}(x, y, z, T) \frac{\partial \Phi_{V}(x, y, z, t)}{\partial x}\right] \\
& +\frac{\partial}{\partial y}\left[D_{\Phi_{V}}(x, y, z, T) \frac{\partial \Phi_{V}(x, y, z, t)}{\partial y}\right] \\
& +\frac{\partial}{\partial z}\left[D_{\Phi_{V}}(x, y, z, T) \frac{\partial \Phi_{V}(x, y, z, t)}{\partial z}\right] \\
& +k_{V, V}(x, y, z, T) V^{2}(x, y, z, t) \\
& -k_{V}(x, y, z, T) V(x, y, z, t) \\
& +\frac{\partial}{\partial x}\left[\frac{D_{\Phi_{V} s}}{\bar{V} k T} \frac{\partial \mu(x, y, z, t)}{\partial x}\right] \\
& +\frac{\partial}{\partial y}\left[\frac{D_{\Phi_{V} s}}{\bar{V} k T} \frac{\partial \mu(x, y, z, t)}{\partial y}\right] \\
& +\frac{\partial}{\partial z}\left[\frac{D_{\Phi_{V} S}}{\bar{V} k T} \frac{\partial \mu(x, y, z, t)}{\partial z}\right] \\
& +f_{\Phi_{I}}(x, y, z) \delta(t) \text {. }
\end{aligned}
$$

Farther, we replace the required concentrations in right sides of (1a) and (3a) on their not yet known average values $\alpha_{1 \rho}$. The replacement gives us possibility to obtain the following equations for determining the first-order approximations of concentrations of radiation defects in the following form:

$$
\begin{aligned}
\frac{\partial I_{1}(x, y, z, t)}{\partial t}= & \frac{\partial}{\partial x}\left[\frac{D_{I S}}{\bar{V} k T} \frac{\partial \mu_{2}(x, y, z, t)}{\partial x}\right] \\
& +\frac{\partial}{\partial y}\left[\frac{D_{I S}}{\bar{V} k T} \frac{\partial \mu_{2}(x, y, z, t)}{\partial y}\right] \\
& +f_{I}(x, y, z) \delta(t) \\
& +\frac{\partial}{\partial z}\left[\frac{D_{I S}}{\bar{V} k T} \frac{\partial \mu_{2}(x, y, z, t)}{\partial z}\right] \\
& -\alpha_{1 I}^{2} k_{I, I}(x, y, z, T) \\
& -\alpha_{1 I} \alpha_{1 V} k_{I, V}(x, y, z, T), \\
\frac{\partial V_{1}(x, y, z, t)}{\partial t}= & \frac{\partial}{\partial x}\left[\frac{D_{V S}}{\bar{V} k T} \frac{\partial \mu_{2}(x, y, z, t)}{\partial x}\right] \\
& +\frac{\partial}{\partial y}\left[\frac{D_{V S}}{\bar{V} k T} \frac{\partial \mu_{2}(x, y, z, t)}{\partial y}\right]
\end{aligned}
$$

$$
\begin{aligned}
& +f_{V}(x, y, z) \delta(t) \\
& +\frac{\partial}{\partial z}\left[\frac{D_{V S}}{\bar{V} k T} \frac{\partial \mu_{2}(x, y, z, t)}{\partial z}\right] \\
& -\alpha_{1 V}^{2} k_{V, V}(x, y, z, T) \\
& -\alpha_{1 I} \alpha_{1 V} k_{I, V}(x, y, z, T),
\end{aligned}
$$

$$
\begin{aligned}
& \frac{\partial \Phi_{1 I}(x, y, z, t)}{\partial t}=\frac{\partial}{\partial x}\left[\frac{D_{\Phi_{I} S}}{\bar{V} k T} \frac{\partial \mu_{2}(x, y, z, t)}{\partial x}\right] \\
& +\frac{\partial}{\partial y}\left[\frac{D_{\Phi_{I} S}}{\bar{V} k T} \frac{\partial \mu_{2}(x, y, z, t)}{\partial y}\right] \\
& +f_{\Phi_{I}}(x, y, z) \delta(t) \\
& +\frac{\partial}{\partial z}\left[\frac{D_{\Phi_{I} S}}{\bar{V} k T} \frac{\partial \mu_{2}(x, y, z, t)}{\partial z}\right] \\
& +k_{I}(x, y, z, T) I(x, y, z, t) \\
& +k_{I, I}(x, y, z, T) I^{2}(x, y, z, t), \\
& \frac{\partial \Phi_{1 V}(x, y, z, t)}{\partial t}=\frac{\partial}{\partial x}\left[\frac{D_{\Phi_{V} S}}{\bar{V} k T} \frac{\partial \mu_{2}(x, y, z, t)}{\partial x}\right] \\
& +\frac{\partial}{\partial y}\left[\frac{D_{\Phi_{V} S}}{\bar{V} k T} \frac{\partial \mu_{2}(x, y, z, t)}{\partial y}\right] \\
& +f_{\Phi_{V}}(x, y, z) \delta(t) \\
& +\frac{\partial}{\partial z}\left[\frac{D_{\Phi_{V} S}}{\bar{V} k T} \frac{\partial \mu_{2}(x, y, z, t)}{\partial z}\right] \\
& +k_{V}(x, y, z, T) V(x, y, z, t) \\
& +k_{V, V}(x, y, z, T) V^{2}(x, y, z, t) .
\end{aligned}
$$

Integration of the left and right sides of (1b) and (3b) on time gives a possibility to obtain the first-order approximations of concentrations of radiation defects in the final form:

$$
\begin{aligned}
& I_{1}(x, y, z, t) \\
& =\frac{\partial}{\partial x} \int_{0}^{t} \frac{D_{I S}}{\bar{V} k T} \frac{\partial \mu_{2}(x, y, z, t)}{\partial x} d \tau \\
& \quad+\frac{\partial}{\partial y} \int_{0}^{t} \frac{D_{I S}}{\bar{V} k T} \frac{\partial \mu_{2}(x, y, z, t)}{\partial y} d \tau+f_{I}(x, y, z) \\
& \quad+\frac{\partial}{\partial z} \int_{0}^{t} \frac{D_{I S}}{\bar{V} k T} \frac{\partial \mu_{2}(x, y, z, t)}{\partial z} d \tau \\
& \quad-\alpha_{1 I}^{2} \int_{0}^{t} k_{I, I}(x, y, z, T) d \tau \\
& \quad-\alpha_{1 I} \alpha_{1 V} \int_{0}^{t} k_{I, V}(x, y, z, T) d \tau,
\end{aligned}
$$




$$
\begin{aligned}
& V_{1}(x, y, z, t) \\
& =\frac{\partial}{\partial x} \int_{0}^{t} \frac{D_{V S}}{\bar{V} k T} \frac{\partial \mu_{2}(x, y, z, t)}{\partial x} d \tau \\
& \quad+\frac{\partial}{\partial y} \int_{0}^{t} \frac{D_{V S}}{\bar{V} k T} \frac{\partial \mu_{2}(x, y, z, t)}{\partial y} d \tau+f_{V}(x, y, z) \\
& \quad+\frac{\partial}{\partial z} \int_{0}^{t} \frac{D_{V S}}{\bar{V} k T} \frac{\partial \mu_{2}(x, y, z, t)}{\partial z} d \tau \\
& \quad-\alpha_{1 V}^{2} \int_{0}^{t} k_{V, V}(x, y, z, T) d \tau \\
& \quad-\alpha_{1 I} \alpha_{1 V} \int_{0}^{t} k_{I, V}(x, y, z, T) d \tau
\end{aligned}
$$$$
\Phi_{1 I}(x, y, z, t)
$$$$
=f_{\Phi_{I}}(x, y, z)+\int_{0}^{t} k_{I}(x, y, z, T) I(x, y, z, \tau) d \tau
$$$$
+\int_{0}^{t} k_{I, I}(x, y, z, T) I^{2}(x, y, z, \tau) d \tau
$$$$
+\frac{\partial}{\partial x} \int_{0}^{t} \frac{D_{\Phi_{I} S}}{\bar{V} k T} \frac{\partial \mu_{2}(x, y, z, \tau)}{\partial x} d \tau
$$$$
+\frac{\partial}{\partial y} \int_{0}^{t} \frac{D_{\Phi_{I} S}}{\bar{V} k T} \frac{\partial \mu_{2}(x, y, z, \tau)}{\partial y} d \tau
$$$$
+\frac{\partial}{\partial z} \int_{0}^{t} \frac{D_{\Phi_{I} S}}{\bar{V} k T} \frac{\partial \mu_{2}(x, y, z, \tau)}{\partial z} d \tau
$$$$
\Phi_{1 V}(x, y, z, t)
$$

$$
\begin{aligned}
= & f_{\Phi_{V}}(x, y, z)+\int_{0}^{t} k_{V}(x, y, z, T) V(x, y, z, \tau) d \tau \\
& +\int_{0}^{t} k_{V, V}(x, y, z, T) V^{2}(x, y, z, \tau) d \tau \\
& +\frac{\partial}{\partial x} \int_{0}^{t} \frac{D_{\Phi_{V} S}}{\bar{V} k T} \frac{\partial \mu_{2}(x, y, z, \tau)}{\partial x} d \tau
\end{aligned}
$$

$$
\begin{aligned}
& +\frac{\partial}{\partial y} \int_{0}^{t} \frac{D_{\Phi_{V} S}}{\bar{V} k T} \frac{\partial \mu_{2}(x, y, z, \tau)}{\partial y} d \tau \\
& +\frac{\partial}{\partial z} \int_{0}^{t} \frac{D_{\Phi_{V} s}}{\bar{V} k T} \frac{\partial \mu_{2}(x, y, z, \tau)}{\partial z} d \tau
\end{aligned}
$$

Average values of the first-order approximations of the required approximations could be calculated by the following relation [14-16]:

$$
\begin{aligned}
\alpha_{1 \rho}= & \frac{1}{\Theta L_{x} L_{y} L_{z}} \\
& \cdot \int_{0}^{\Theta} \int_{0}^{L_{x}} \int_{0}^{L_{y}} \int_{0}^{L_{z}} \rho_{1}(x, y, z, t) d z d y d x d t .
\end{aligned}
$$

Substitution of relations (1c) and (3c) into relation (6) gives a possibility to calculate the required average values in the following form:

$$
\begin{aligned}
\alpha_{1 I} & =\sqrt{\frac{\left(a_{3}+A\right)^{2}}{4 a_{4}^{2}}-4\left(B+\frac{\Theta a_{3} B+\Theta^{2} L_{x} L_{y} L_{z} a_{1}}{a_{4}}\right)} \\
& -\frac{a_{3}+A}{4 a_{4}}, \\
\alpha_{1 V} & =\frac{1}{S_{I V 00}}\left[\frac{\Theta}{\alpha_{1 I}} \int_{0}^{L_{x}} \int_{0}^{L_{y}} \int_{0}^{L_{z}} f_{I}(x, y, z) d z d y d x\right. \\
- & \left.\alpha_{1 I} S_{I I 00}-\Theta L_{x} L_{y} L_{z}\right], \\
\alpha_{1 \Phi_{I}} & =\frac{R_{I 1}}{\Theta L_{x} L_{y} L_{z}}+\frac{S_{I I 20}}{\Theta L_{x} L_{y} L_{z}}+\frac{1}{L_{x} L_{y} L_{z}} \\
& \cdot \int_{0}^{L_{x}} \int_{0}^{L_{y}} \int_{0}^{L_{z}} f_{\Phi_{I}}(x, y, z) d z d y d x, \\
& +\frac{\left(R_{V 1}+S_{V V 20}\right)}{\Theta L_{x} L_{y} L_{z}} . \\
\alpha_{1 \Phi_{V}} & =\frac{1}{L_{x} L_{y} L_{z}} \int_{0}^{L_{x}} \int_{0}^{L_{y}} \int_{0}^{L_{z}} f_{\Phi_{V}}(x, y, z) d z d y d x
\end{aligned}
$$

Here,

$$
\begin{aligned}
S_{\rho \rho i j}= & \int_{0}^{\Theta}(\Theta-t) \int_{0}^{L_{x}} \int_{0}^{L_{y}} \int_{0}^{L_{z}} k_{\rho, \rho}(x, y, z, T) I_{1}^{i}(x, y, z, t) V_{1}^{j}(x, y, z, t) d z d y d x d t, \\
a_{4}= & S_{I I 00}\left(S_{I V 00}^{2}-S_{I I 00} S_{V V 00}\right), \\
a_{3}= & S_{I V 00} S_{I I 00}+S_{I V 00}^{2}-S_{I I 00} S_{V V 00}, \\
a_{2}= & \int_{0}^{L_{x}} \int_{0}^{L_{y}} \int_{0}^{L_{z}} f_{V}(x, y, z) d z d y d x S_{I V 00} S_{I V 00}^{2}+2 S_{V V 00} S_{I I 00} \int_{0}^{L_{x}} \int_{0}^{L_{y}} \int_{0}^{L_{z}} f_{I}(x, y, z) d z d y d x \\
& -S_{I V 00}^{2} \int_{0}^{L_{x}} \int_{0}^{L_{y}} \int_{0}^{L_{z}} f_{I}(x, y, z) d z d y d x+S_{I V 00} \Theta L_{x}^{2} L_{y}^{2} L_{z}^{2}-\Theta L_{x}^{2} L_{y}^{2} L_{z}^{2} S_{V V 00},
\end{aligned}
$$




$$
\begin{aligned}
a_{1} & =S_{I V 00} \int_{0}^{L_{x}} \int_{0}^{L_{y}} \int_{0}^{L_{z}} f_{I}(x, y, z) d z d y d x \\
A & =\sqrt{8 y+\Theta^{2} \frac{a_{3}^{2}}{a_{4}^{2}}-4 \Theta \frac{a_{2}}{a_{4}}}, \\
a_{0} & =S_{V V 00}\left[\int_{0}^{L_{x}} \int_{0}^{L_{y}} \int_{0}^{L_{z}} f_{I}(x, y, z) d z d y d x\right]^{2}, \\
B & =\frac{\Theta a_{2}}{6 a_{4}}+\sqrt[3]{\sqrt{q^{2}+p^{3}}-q}-\sqrt[3]{\sqrt{q^{2}+p^{3}}+q}, \\
q & =\frac{\Theta^{3} a_{2}}{24 a_{4}^{2}}\left(4 a_{0}-\Theta L_{x} L_{y} L_{z} \frac{a_{1} a_{3}}{a_{4}}\right)-\Theta^{2} \frac{a_{0}}{8 a_{4}^{2}}\left(4 \Theta a_{2}-\Theta^{2} \frac{a_{3}^{2}}{a_{4}}\right)-\frac{\Theta^{3} a_{2}^{3}}{54 a_{4}^{3}}-L_{x}^{2} L_{y}^{2} L_{z}^{2} \frac{\Theta^{4} a_{1}^{2}}{8 a_{4}^{2}}, \\
R_{\rho i} & =\int_{0}^{\Theta}(\Theta-t) \int_{0}^{L_{x}} \int_{0}^{L_{y}} \int_{0}^{L_{z}} k_{I}(x, y, z, T) I_{1}^{i}(x, y, z, t) d z d y d x d t, \\
p & =\Theta^{2} \frac{4 a_{0} a_{4}}{12 a_{4}^{2}}-\frac{\Theta a_{2}}{18 a_{4}}-\frac{\Theta a_{1} a_{3}}{12 a_{4}^{2}} L_{x} L_{y} L_{z} .
\end{aligned}
$$

We determine approximations with the second and higher orders of concentrations of radiations defects framework standard iterative procedure of method of averaging of function corrections [14-16]. For the framework of the procedure, we determine the approximation of $n$th order by replacement of the concentrations of radiation defects $I(x, y, z, t), V(x, y, z, t), \Phi_{I}(x, y, z, t)$, and $\Phi_{V}(x, y, z, t)$ in right sides of (1b) and (3b) on the following sums $\alpha_{n \rho}+$ $\rho_{n-1}(x, y, z, t)$. The replacement gives a possibility to obtain the second-order approximations of concentrations of radiation defects:

$$
\begin{aligned}
& \frac{\partial I_{2}(x, y, z, t)}{\partial t}=\frac{\partial}{\partial x}\left[D_{I}(x, y, z, T) \frac{\partial I_{1}(x, y, z, t)}{\partial x}\right] \\
& +\frac{\partial}{\partial y}\left[D_{I}(x, y, z, T) \frac{\partial I_{1}(x, y, z, t)}{\partial y}\right] \\
& +\frac{\partial}{\partial z}\left[D_{I}(x, y, z, T) \frac{\partial I_{1}(x, y, z, t)}{\partial z}\right] \\
& -k_{I, V}(x, y, z, T)\left[\alpha_{1 I}+I_{1}(x, y, z, t)\right] \\
& \cdot\left[\alpha_{1 V}+V_{1}(x, y, z, t)\right]+\frac{\partial}{\partial x} \\
& \cdot \int_{0}^{t} \frac{D_{I S}}{\bar{V} k T} \frac{\partial \mu_{2}(x, y, z, t)}{\partial x} d \tau+\frac{\partial}{\partial y} \\
& \cdot \int_{0}^{t} \frac{D_{I S}}{\bar{V} k T} \frac{\partial \mu_{2}(x, y, z, t)}{\partial y} d \tau+\frac{\partial}{\partial z} \\
& \cdot \int_{0}^{t} \frac{D_{I S}}{\bar{V} k T} \frac{\partial \mu_{2}(x, y, z, t)}{\partial z} d \tau-k_{I, I}(x, y, z, T) \\
& \cdot\left[\alpha_{1 I}+I_{1}(x, y, z, t)\right]^{2},
\end{aligned}
$$

$$
\begin{aligned}
& \frac{\partial V_{2}(x, y, z, t)}{\partial t} \\
& =\frac{\partial}{\partial x}\left[D_{V}(x, y, z, T) \frac{\partial V_{1}(x, y, z, t)}{\partial x}\right] \\
& +\frac{\partial}{\partial y}\left[D_{V}(x, y, z, T) \frac{\partial V_{1}(x, y, z, t)}{\partial y}\right] \\
& +\frac{\partial}{\partial z}\left[D_{V}(x, y, z, T) \frac{\partial V_{1}(x, y, z, t)}{\partial z}\right] \\
& -k_{I, V}(x, y, z, T)\left[\alpha_{1 I}+I_{1}(x, y, z, t)\right] \\
& \cdot\left[\alpha_{1 V}+V_{1}(x, y, z, t)\right]+\frac{\partial}{\partial x} \\
& \cdot \int_{0}^{t} \frac{D_{I S}}{\bar{V} k T} \frac{\partial \mu_{2}(x, y, z, t)}{\partial x} d \tau+\frac{\partial}{\partial y} \\
& \cdot \int_{0}^{t} \frac{D_{I S}}{\bar{V} k T} \frac{\partial \mu_{2}(x, y, z, t)}{\partial y} d \tau+\frac{\partial}{\partial z} \\
& \cdot \int_{0}^{t} \frac{D_{I S}}{\bar{V} k T} \frac{\partial \mu_{2}(x, y, z, t)}{\partial z} d \tau-k_{V, V}(x, y, z, T) \\
& \cdot\left[\alpha_{1 I}+V_{1}(x, y, z, t)\right]^{2}, \\
& \frac{\partial \Phi_{2 I}(x, y, z, t)}{\partial t} \\
& =\frac{\partial}{\partial x}\left[D_{\Phi_{I}}(x, y, z, T) \frac{\partial \Phi_{1 I}(x, y, z, t)}{\partial x}\right]
\end{aligned}
$$




$$
\begin{aligned}
& +\frac{\partial}{\partial y}\left[D_{\Phi_{I}}(x, y, z, T) \frac{\partial \Phi_{1 I}(x, y, z, t)}{\partial y}\right] \\
& +\frac{\partial}{\partial z}\left[D_{\Phi_{I}}(x, y, z, T) \frac{\partial \Phi_{1 I}(x, y, z, t)}{\partial z}\right] \\
& +k_{I, I}(x, y, z, T) I^{2}(x, y, z, t)+k_{I}(x, y, z, T) \\
& +I(x, y, z, t)+\frac{\partial}{\partial x}\left[\frac{D_{\Phi_{I} S}}{\bar{V} k T} \frac{\partial \mu_{2}(x, y, z, t)}{\partial x}\right] \\
& +\frac{\partial}{\partial y}\left[\frac{D_{\Phi_{I} S}}{\bar{V} k T} \frac{\partial \mu_{2}(x, y, z, t)}{\partial y}\right] \\
& +\frac{\partial}{\partial z}\left[\frac{D_{\Phi_{I} S}}{\bar{V} k T} \frac{\partial \mu_{2}(x, y, z, t)}{\partial z}\right]+f_{\Phi_{I}}(x, y, z) \delta(t) \\
& +\frac{\partial}{\partial V}\left[\frac { \partial } { \overline { V } k T } \left[\frac{D_{\Phi_{V} S}}{\bar{V} k T} \frac{\partial \mu_{2}(x, y, z, t)}{\partial y, t)}\right.\right. \\
& \left.+\frac{\partial}{\partial t}\right] \\
& +\frac{\partial}{\partial z}[x, y, z, t)+\frac{\partial}{\partial x}\left[\frac{D_{\Phi_{V} S}}{\bar{V}} \frac{\partial \mu_{2}(x, y, z, t)}{\partial x}\right]+f_{\Phi_{V}}(x, y, z) \delta(t) \\
& +
\end{aligned}
$$

Integration of left and right sides of (1d) and ( $3 \mathrm{~d})$ gives a possibility to obtain relations for the second-order approximations of the required concentrations of radiation defects in the following form:

$$
\begin{aligned}
& I_{2}(x, y, z, t)=\frac{\partial}{\partial x} \int_{0}^{t} D_{I}(x, y, z, T) \frac{\partial I_{1}(x, y, z, \tau)}{\partial x} d \tau \\
& \quad+\frac{\partial}{\partial y} \int_{0}^{t} D_{I}(x, y, z, T) \frac{\partial I_{1}(x, y, z, \tau)}{\partial y} d \tau+\frac{\partial}{\partial z} \\
& \quad \cdot \int_{0}^{t} D_{I}(x, y, z, \\
& \quad T) \frac{\partial I_{1}(x, y, z, \tau)}{\partial z} d \tau \\
& \quad-\int_{0}^{t} k_{I, I}(x, y, z, T)\left[\alpha_{2 I}+I_{1}(x, y, z, \tau)\right]^{2} d \tau
\end{aligned}
$$

$-\int_{0}^{t} k_{I, V}(x, y, z, T)\left[\alpha_{2 I}+I_{1}(x, y, z, \tau)\right]\left[\alpha_{2 V}\right.$

$\left.+V_{1}(x, y, z, \tau)\right] d \tau+f_{I}(x, y, z)$

$+\frac{\partial}{\partial x}\left[\frac{D_{I S}}{\bar{V} k T} \frac{\partial \mu_{2}(x, y, z, t)}{\partial x}\right]+\frac{\partial}{\partial y}\left[\frac{D_{I S}}{\bar{V} k T}\right.$

$\left.\frac{\partial \mu_{2}(x, y, z, t)}{\partial y}\right]+\frac{\partial}{\partial z}\left[\frac{D_{I S}}{\bar{V} k T} \frac{\partial \mu_{2}(x, y, z, t)}{\partial z}\right]$,

$$
\begin{aligned}
& V_{2}(x, y, z, t)=\frac{\partial}{\partial x} \int_{0}^{t} D_{V}(x, y, z, \\
& \text { T) } \frac{\partial V_{1}(x, y, z, \tau)}{\partial x} d \tau \\
& +\frac{\partial}{\partial y} \int_{0}^{t} D_{V}(x, y, z, T) \frac{\partial V_{1}(x, y, z, \tau)}{\partial y} d \tau+\frac{\partial}{\partial z} \\
& \cdot \int_{0}^{t} D_{V}(x, y, z \\
& \text { T) } \frac{\partial V_{1}(x, y, z, \tau)}{\partial z} d \tau \\
& -\int_{0}^{t} k_{V, V}(x, y, z, T)\left[\alpha_{2 V}+V_{1}(x, y, z, \tau)\right]^{2} d \tau \\
& -\int_{0}^{t} k_{I, V}(x, y, z, T)\left[\alpha_{V I}+I_{1}(x, y, z, \tau)\right]\left[\alpha_{2 V}\right. \\
& \left.+V_{1}(x, y, z, \tau)\right] d \tau+f_{V}(x, y, z) \\
& +\frac{\partial}{\partial x}\left[\frac{D_{V S}}{\bar{V} k T} \frac{\partial \mu_{2}(x, y, z, t)}{\partial x}\right]+\frac{\partial}{\partial y}\left[\frac{D_{V S}}{\bar{V} k T}\right. \\
& \left.\frac{\partial \mu_{2}(x, y, z, t)}{\partial y}\right]+\frac{\partial}{\partial z}\left[\frac{D_{V S}}{\bar{V} k T} \frac{\partial \mu_{2}(x, y, z, t)}{\partial z}\right] \text {, }
\end{aligned}
$$$$
\Phi_{2 I}(x, y, z, t)=\frac{\partial}{\partial x} \int_{0}^{t} D_{\Phi_{I}}(x, y, z
$$$$
\text { T) } \frac{\partial \Phi_{1 I}(x, y, z, \tau)}{\partial x} d \tau
$$$$
+f_{\Phi_{I}}(x, y, z)+\frac{\partial}{\partial y} \int_{0}^{t} D_{\Phi_{I}}(x, y, z,
$$$$
\text { T) } \frac{\partial \Phi_{1 I}(x, y, z, \tau)}{\partial y} d \tau
$$$$
+\frac{\partial}{\partial z} \int_{0}^{t} D_{\Phi_{I}}(x, y, z, T) \frac{\partial \Phi_{1 I}(x, y, z, \tau)}{\partial z} d \tau+\frac{\partial}{\partial x}
$$$$
\cdot \int_{0}^{t} \frac{D_{\Phi_{I} S}}{\bar{V} k T} \frac{\partial \mu_{2}(x, y, z, \tau)}{\partial x} d \tau+\frac{\partial}{\partial y}
$$$$
\cdot \int_{0}^{t} \frac{D_{\Phi_{I} S}}{\bar{V} k T} \frac{\partial \mu_{2}(x, y, z, \tau)}{\partial y} d \tau+\int_{0}^{t} k_{I, I}(x, y, z, T)
$$ 


$$
\begin{aligned}
& \times I^{2}(x, y, z, \tau) d \tau+\frac{\partial}{\partial z} \\
& \int_{0}^{t} \frac{D_{\Phi_{I} S}}{\bar{V} k T} \frac{\partial \mu_{2}(x, y, z, \tau)}{\partial z} d \tau+\int_{0}^{t} k_{I}(x, y, z, T) \\
\cdot & I(x, y, z, \tau) d \tau \\
\Phi_{2 V} & (x, y, z, t)=\frac{\partial}{\partial x} \int_{0}^{t} D_{\Phi_{V}}(x, y, z, \\
T) & \frac{\partial \Phi_{1 V}(x, y, z, \tau)}{\partial x} d \tau \\
+ & f_{\Phi_{V}}(x, y, z)+\frac{\partial}{\partial y} \int_{0}^{t} D_{\Phi_{V}}(x, y, z, T) \\
& \times \frac{\partial \Phi_{1 V}(x, y, z, \tau)}{\partial y} d \tau+\frac{\partial}{\partial z} \int_{0}^{t} D_{\Phi_{V}}(x, y, z, \\
& T) \frac{\partial \Phi_{1 V}(x, y, z, \tau)}{\partial z} d \tau \\
+ & \int_{0}^{t} k_{V}(x, y, z, T) V(x, y, z, \tau) d \tau+\frac{\partial}{\partial x}
\end{aligned}
$$

$$
\begin{aligned}
& \cdot \int_{0}^{t} \frac{D_{\Phi_{V} S}}{\bar{V} k T} \frac{\partial \mu_{2}(x, y, z, \tau)}{\partial x} d \tau+\frac{\partial}{\partial y} \\
& \cdot \int_{0}^{t} \frac{D_{\Phi_{V} S}}{\bar{V} k T} \frac{\partial \mu_{2}(x, y, z, \tau)}{\partial y} d \tau+\frac{\partial}{\partial z} \\
& \cdot \int_{0}^{t} \frac{D_{\Phi_{V} S}}{\bar{V} k T} \frac{\partial \mu_{2}(x, y, z, \tau)}{\partial z} d \tau+\int_{0}^{t} k_{V, V}(x, y, z, T) \\
& \cdot V^{2}(x, y, z, \tau) d \tau .
\end{aligned}
$$

We determine average values of the second-order approximations by the standard relation [14-16]:

$$
\begin{aligned}
\alpha_{2 \rho} & =\frac{1}{\Theta L_{x} L_{y} L_{z}} \\
& \cdot \int_{0}^{\Theta} \int_{0}^{L_{x}} \int_{0}^{L_{y}} \int_{0}^{L_{z}}\left[\rho_{2}(x, y, z, t)-\rho_{1}(x, y, z, t)\right] d z d y d x d t .
\end{aligned}
$$

Substitution of relations (1e) and (3e) in relation (9) gives a possibility to obtain relations for the required values $\alpha_{2 \rho}$ :

$$
\begin{aligned}
\alpha_{2 C} & =0, \\
\alpha_{2 \Phi_{I}} & =0, \\
\alpha_{2 \Phi_{V}} & =0 \\
\alpha_{2 V} & =\sqrt{\frac{\left(b_{3}+E\right)^{2}}{4 b_{4}^{2}}-4\left(F+\frac{\Theta a_{3} F+\Theta^{2} L_{x} L_{y} L_{z} b_{1}}{b_{4}}\right)}-\frac{b_{3}+E}{4 b_{4}}, \\
\alpha_{2 I} & =\frac{C_{V}-\alpha_{2 V}^{2} S_{V V 00}-\alpha_{2 V}\left(2 S_{V V 01}+S_{I V 10}+\Theta L_{x} L_{y} L_{z}\right)-S_{V V 02}-S_{I V 11}}{S_{I V 01}+\alpha_{2 V} S_{I V 00}},
\end{aligned}
$$

where

$$
\begin{aligned}
b_{4}= & \frac{1}{\Theta L_{x} L_{y} L_{z}} S_{I V 00}^{2} S_{V V 00}-\frac{1}{\Theta L_{x} L_{y} L_{z}} S_{V V 00}^{2} S_{I I 00}, \\
b_{3}= & -\left(2 S_{V V 01}+S_{I V 10}+\Theta L_{x} L_{y} L_{z}\right) \frac{S_{I I 00} S_{V V 00}}{\Theta L_{x} L_{y} L_{z}} \\
& +\frac{S_{I V 00} S_{V V 00}}{\Theta L_{x} L_{y} L_{z}}\left(S_{I V 01}+2 S_{I I 10}+S_{I V 01}+\Theta L_{x} L_{y} L_{z}\right) \\
& +\left(2 S_{V V 01}+S_{I V 10}+\Theta L_{x} L_{y} L_{z}\right) \frac{S_{I V 00}^{2}}{\Theta L_{x}} \frac{1}{L_{y} L_{z}} \\
& -\frac{S_{I V 00}^{2} S_{I V 10}}{\Theta^{3} L_{x}^{3} L_{y}^{3} L_{z}^{3}}, \\
b_{2} & =\frac{S_{I I 00} S_{V V 00}}{\Theta L_{x} L_{y} L_{z}}\left(S_{V V 02}+S_{I V 11}+C_{V}\right)-\left(\Theta L_{x} L_{y} L_{z}\right.
\end{aligned}
$$

$$
\begin{aligned}
- & \left.2 S_{V V 01}+S_{I V 10}\right)^{2}+\frac{S_{I V 01} S_{V V 00}}{\Theta L_{x} L_{y} L_{z}}\left(\Theta L_{x} L_{y} L_{z}\right. \\
+ & \left.2 S_{I I 10}+S_{I V 01}\right)+\frac{S_{I V 00}}{\Theta L_{x} L_{y} L_{z}}\left(\Theta L_{x} L_{y} L_{z}+S_{I V 01}\right. \\
+ & \left.2 S_{I I 10}+2 S_{I V 01}\right)\left(\Theta L_{x} L_{y} L_{z}+2 S_{V V 01}+S_{I V 10}\right) \\
- & \frac{C_{V}-S_{V V 02}-S_{I V 11}}{\Theta L_{x} L_{y} L_{z}}+\frac{C_{I} S_{I V 00}^{2}}{\Theta L_{x}^{2} L_{y}^{2} L_{z}^{2}}-2 S_{I V 10} \\
& \cdot \frac{S_{I V 00} S_{I V 01}}{\Theta L_{x} L_{y} L_{z}}, \\
b_{1} & S_{I I 00} \frac{S_{I V 11}+S_{V V 02}+C_{V}}{\Theta L_{x} L_{y} L_{z}}\left(2 S_{V V 01}+S_{I V 10}\right. \\
+ & \left.\Theta L_{x} L_{y} L_{z}\right)+\frac{S_{I V 01}}{\Theta L_{x} L_{y} L_{z}}\left(\Theta L_{x} L_{y} L_{z}+2 S_{I I 10}\right.
\end{aligned}
$$




$$
\begin{aligned}
& \left.+S_{I V 01}\right)\left(2 S_{V V 01}+S_{I V 10}+\Theta L_{x} L_{y} L_{z}\right) \\
& -\frac{S_{I V 00}}{\Theta L_{x} L_{y} L_{z}}\left(3 S_{I V 01}+2 S_{I I 10}+\Theta L_{x} L_{y} L_{z}\right)\left(C_{V}\right. \\
& \left.-S_{V V 02}-S_{I V 11}\right)+2 C_{I} S_{I V 00} S_{I V 01}-\frac{S_{I V 10} S_{I V 01}^{2}}{\Theta L_{x} L_{y} L_{z}}, \\
& b_{0}=\frac{S_{I I 00}}{\Theta L_{x} L_{y} L_{z}}\left(S_{I V 00}+S_{V V 02}\right)^{2} \\
& -\frac{S_{I V 01}}{\Theta L_{x} L_{y} L_{z}}\left(\Theta L_{x} L_{y} L_{z}+2 S_{I I 10}+S_{I V 01}\right)\left(C_{V}\right. \\
& \left.-S_{V V 02}-S_{I V 11}\right)+2 C_{I} S_{I V 01}^{2}-\frac{C_{V}-S_{V V 02}-S_{I V 11}}{\Theta L_{x} L_{y} L_{z}} \\
& \cdot S_{I V 01}\left(\Theta L_{x} L_{y} L_{z}+2 S_{I I 10}+S_{I V 01}\right) \text {, } \\
& C_{I}=\frac{\alpha_{1 I} \alpha_{1 V}}{\Theta L_{x} L_{y} L_{z}} S_{I V 00}+\frac{\alpha_{1 I}^{2} S_{I I 00}}{\Theta L_{x} L_{y} L_{z}}-\frac{S_{I I 20}}{\Theta L_{x} L_{y} L_{z}} \\
& -\frac{S_{I V 11}}{\Theta L_{x} L_{y} L_{z}}, \\
& C_{V}=\alpha_{1 I} \alpha_{1 V} S_{I V 00}+\alpha_{1 V}^{2} S_{V V 00}-S_{V V 02}-S_{I V 11} \text {, } \\
& E=\sqrt{8 y+\Theta^{2} \frac{a_{3}^{2}}{a_{4}^{2}}-4 \Theta \frac{a_{2}}{a_{4}},} \\
& F=\frac{\Theta a_{2}}{6 a_{4}}+\sqrt[3]{\sqrt{r^{2}+s^{3}}-r}-\sqrt[3]{\sqrt{r^{2}+s^{3}}+r}, \\
& r=\frac{\Theta^{3} b_{2}}{24 b_{4}^{2}}\left(4 b_{0}-\Theta L_{x} L_{y} L_{z} \frac{b_{1} b_{3}}{b_{4}}\right)-b_{0} \frac{\Theta^{2}}{8 b_{4}^{2}}\left(4 \Theta b_{2}\right. \\
& \left.-\Theta^{2} \frac{b_{3}^{2}}{b_{4}}\right)-\frac{\Theta^{3} b_{2}^{3}}{54 b_{4}^{3}}-L_{x}^{2} L_{y}^{2} L_{z}^{2} \frac{\Theta^{4} b_{1}^{2}}{8 b_{4}^{2}}, \\
& s=-\frac{\Theta b_{2}}{18 b_{4}}+\frac{\Theta^{2}\left(4 b_{0} b_{4}-\Theta L_{x} L_{y} L_{z} b_{1} b_{3}\right)}{12 b_{4}^{2}} .
\end{aligned}
$$

For the framework of this paper, the required spatiotemporal distributions of concentrations of radiations defects have been determined by using the second-order approximations by using method of averaging of function corrections. The approximations are usually good enough to make qualitative analysis and to obtain some quantitative results. All obtained results have been checked by comparison with results of numerical simulations. The results of numerical simulations have been obtained by solving (1) and (4) by using standard explicit difference scheme.

\section{Discussion}

In this section, we analyzed distributions of concentrations of point defects and their simplest complexes. In the previous section, we analytically take into account porosity of

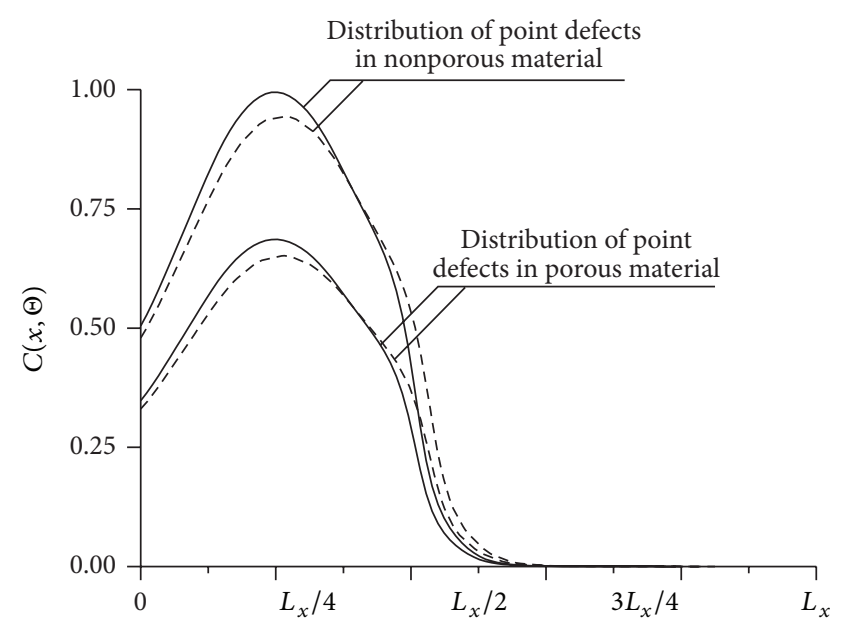

FIGURE 2: Distributions of concentrations of point radiation defects for fixed value of annealing time. Curve 1 corresponds to implantation of ions of dopant through nonporous epitaxial layer. Curve 2 corresponds to implantation of ions of dopant through porous epitaxial layer. Solid lines are the analytical results. Dashed lines are the numerical results.

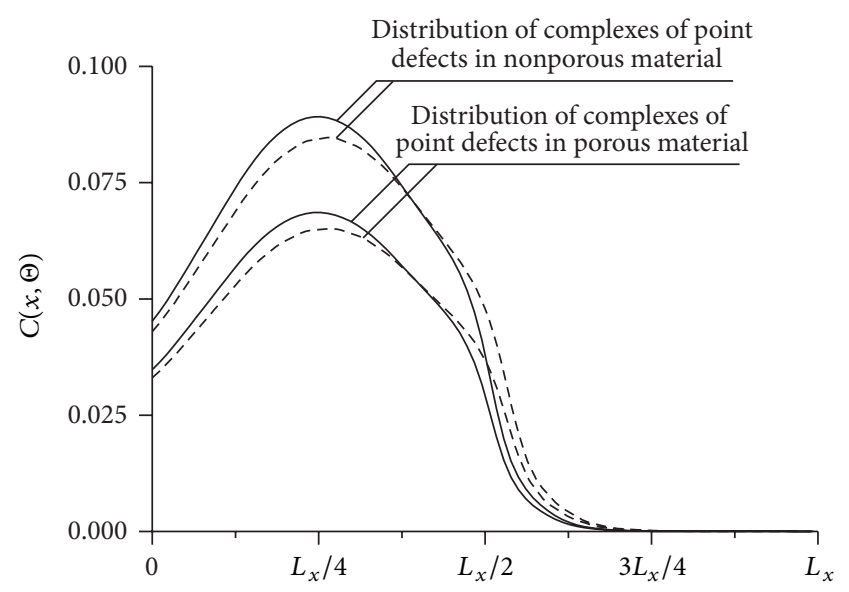

FIgURE 3: Distributions of concentrations of simplest complexes of point radiation defects for fixed value of annealing time. Curve 1 corresponds to implantation of ions of dopant through nonporous epitaxial layer. Curve 2 corresponds to implantation of ions of dopant through porous epitaxial layer. Solid lines are the analytical results. Dashed lines are the numerical results.

materials in comparison with cited similar works. In this situation, we obtain decreasing quantity of radiation defects (one can find decreasing both types of accounted defects: point defects and their simplest complexes) in comparison with nonporous materials. Probably, this effect could be obtained due to draining of these defects to pores. Typical distributions of concentrations of point radiation defects and their simplest complexes are presented in Figures 2 and 3 , respectively. In this situation, using overlayer over device area gives a possibility to increase radiation resistance of the devices during radiation processing. Using porous overlayer gives a possibility to obtain larger increase of radiation resistance. Figures 2 and 3 also show that quantity 
of point defects is larger than quantity of simplest complexes of point defects. This effect could be found because only part of point defects could generate their complexes. It should be also noted that we have also analytically taken into account larger quantity of effects, which could be found during relaxation of distributions of concentrations of radiation defects: diffusion of radiation defects (on account of porosity stimulated diffusion), recombination of defects, and generation and decay of complexes of defects. Analysis of nonlinearity of the relaxation shows that the nonlinearity gives a possibility to decrease quantity of radiation defects after multistage radiation processing in comparison with onestage radiation processing. Distributions of concentrations of radiation defects during comparison of multistage and one-stage radiation processing will be qualitatively similar to distributions in Figures 2 and 3.

\section{Conclusion}

In the present paper, we analyzed redistributions of radiations defects in material with porous and nonporous overlayer after radiation processing. It has been shown that presence of porous overlayer gives a possibility to decrease quantity of radiation defects.

\section{Competing Interests}

The authors declare that the grant, scholarship, and/or funding mentioned in Acknowledgments section do not lead to any competing interests. Additionally, the authors declare that there are no competing interests regarding the publication of this paper.

\section{Acknowledgments}

This work is supported by the Agreement of August 27, 2013, no. 02.B.49.21.0003 between The Ministry of Education and Science of the Russian Federation and Lobachevsky State University of Nizhni Novgorod, educational fellowship for scientific research of Government of Russia, educational fellowship for scientific research of Government of Nizhny Novgorod region of Russia, and educational fellowship for scientific research of Nizhny Novgorod State University of Architecture and Civil Engineering.

\section{References}

[1] A. A. Lebedev, A. M. Ivanov, and N. B. Strokan, "Radiation resistance of $\mathrm{SiC}$ and nuclear-radiation detectors based on $\mathrm{SiC}$ films," Semiconductors, vol. 38, no. 2, pp. 129-150, 2004.

[2] E. V. Kalinina, V. G. Kossov, R. R. Yafaev, A. M. Strel'chuk, and G. N. Violina, "A high-temperature radiation-resistant rectifier based on $\mathrm{p}^{+}-\mathrm{n}$ junctions in $4 \mathrm{H}$-SiC ion-implanted with aluminum," Semiconductors, vol. 44, no. 6, pp. 778-788, 2010.

[3] A. E. Belyaev, N. S. Boltovets, A. V. Bobyl' et al., "Radiation effects and interphase interactions in ohmic and barrier contacts to indium phosphide as induced by rapid thermal annealing and irradiation with $\gamma$-ray ${ }^{60}$ Co photons," Semiconductors, vol. 44, no. 12, pp. 1559-1566, 2010.
[4] G. P. Gaydar, "On the kinetics of electron processes in ${ }^{60} \mathrm{Co} \gamma$ irradiated n-Ge single crystals," Semiconductors, vol. 48, no. 9, pp. 1171-1175, 2014.

[5] P. A. Aleksandrov, N. E. Belova, K. D. Demakov, and S. G. Shemardov, "On the generation of charge-carrier recombination centers in the sapphire substrates of silicon-on-sapphire structures," Semiconductors, vol. 49, no. 8, pp. 1099-1103, 2015.

[6] Y. W. Zhang and A. F. Bower, "Numerical simulations of island formation in a coherent strained epitaxial thin film system," Journal of the Mechanics and Physics of Solids, vol. 47, no. 11, pp. 2273-2297, 1999.

[7] M. Kitayama, T. Narushima, W. C. Carter, R. M. Cannon, and A. M. Glaeser, "Wulff shape of alumina: I, modeling the kinetics of morphological evolution," Journal of the American Ceramic Society, vol. 83, no. 10, pp. 2561-2571, 2000.

[8] M. Kitayama, T. Narushima, and A. M. Glaeser, "Wulff shape of alumina: II, experimental measurements of pore shape evolution rates," Journal of the American Ceramic Society, vol. 83, no. 10, pp. 2572-2583, 2000.

[9] E. L. Pankratov, "Application of porous layers and optimization of annealing of dopant and radiation defects to increase sharpness of p-n-junctions in a bipolar heterotransistors," Journal of Nanoelectronics and Optoelectronics, vol. 6, no. 2, pp. 188-206, 2011.

[10] P. G. Cheremskoy, V. V. Slesov, and V. I. Betekhtin, Pore in Solid Bodies, Energoatomizdat, Moscow, Russia, 1990 (Russian).

[11] V. L. Vinetskiy and G. A. Kholodar', Radiative Physics of Semiconductors, Naukova Dumka, Kiev, Ukraine, 1979 (Russian).

[12] P. M. Fahey, P. B. Griffin, and J. D. Plummer, "Point defects and dopant diffusion in silicon," Reviews of Modern Physics, vol. 61, no. 2, pp. 289-384, 1989.

[13] M. G. Mynbaeva, E. N. Mokhov, A. A. Lavrent'ev, and K. D. Mynbaev, "High-temperature diffusion doping of porous silicon carbide," Technical Physics Letters, vol. 34, no. 9, pp. 731733, 2008.

[14] E. L. Pankratov and E. A. Bulaeva, "About influence of buffer porous layers between epitaxial layers of heterostructure on distributions of concentrations of dopants in heterobipolar transistors," International Journal of Multiphysics, vol. 9, no. 2, pp. 109-136, 2015.

[15] Y. D. Sokolov, "About determination of dynamical forces in mine's hoisting ropes," Applied Mechanics, vol. 1, no. 1, pp. 23-35, 1955.

[16] E. L. Pankratov and E. A. Bulaeva, "Optimization of manufacturing of emitter-coupled logic to decrease surface of chip," International Journal of Modern Physics B, vol. 29, 2015. 

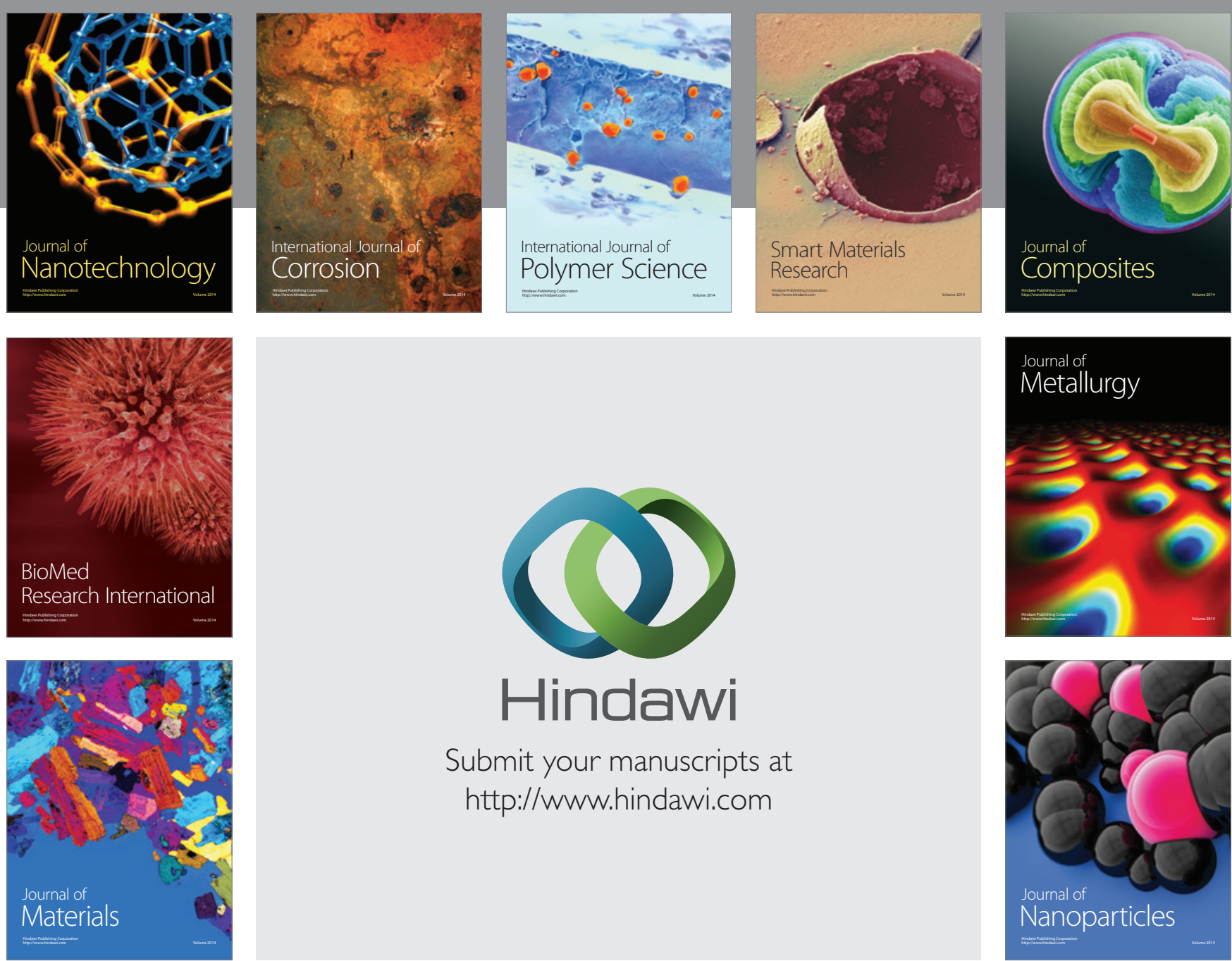

\section{Hindawi}

Submit your manuscripts at

http://www.hindawi.com

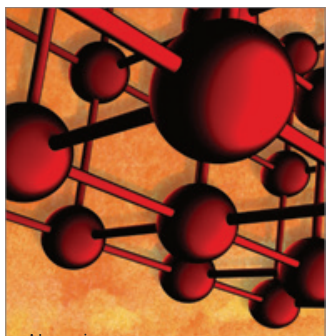

Materials Science and Engineering
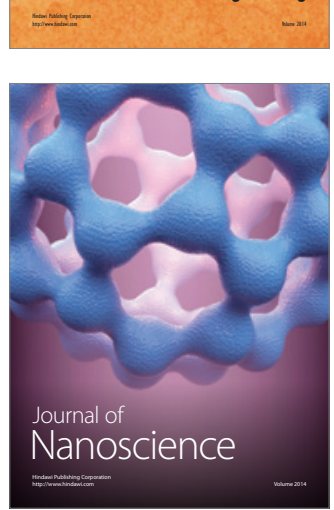
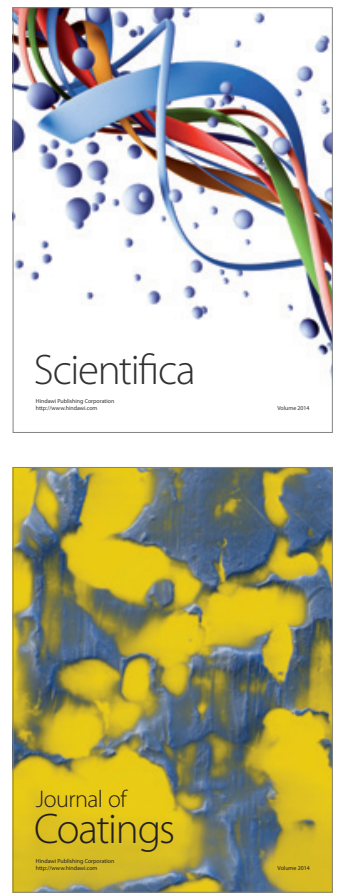
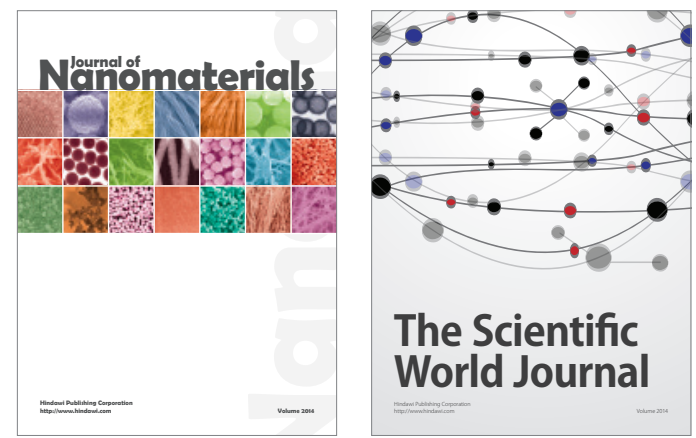

The Scientific World Journal
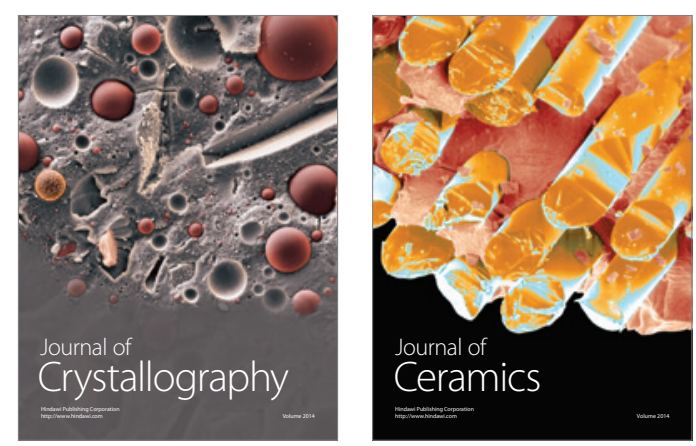
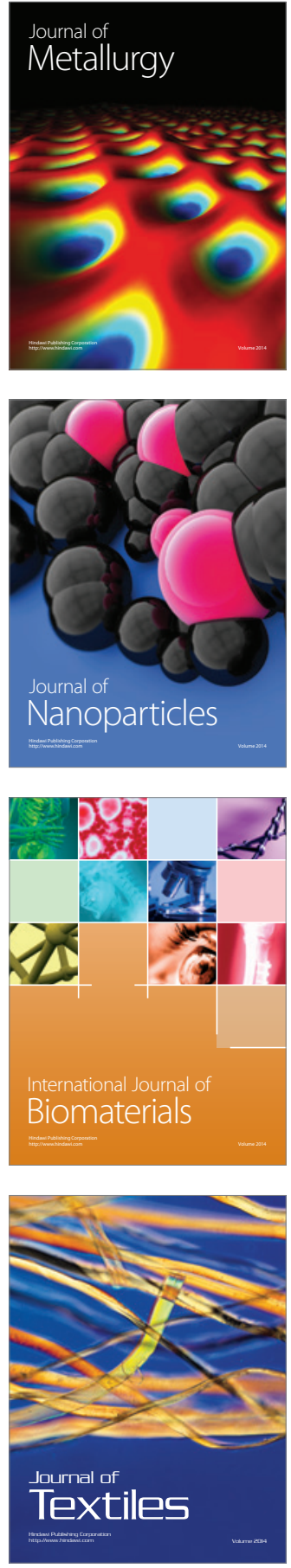